\title{
System Pharmacology-Based Strategy to Investigate Core Component Group and Molecular Mechanisms of Xuefu Zhuyu Decoction in the Treatment of Gbm
}

\section{Zhiying Lin}

Southern Medical University Nanfang Hospital

Kexin Wang

Shanxi University

Runwei Yang

Southern Medical University Nanfang Hospital

Zhiyong Li

Southern Medical University Nanfang Hospital

Guozhong Yi

Southern Medical University Nanfang Hospital

Aiping Lu

Hong Kong Baptist University

Xinghua Pan

Southern Medical University

Yawei Liu

Southern Medical University Nanfang Hospital

Junxiang Peng

Southern Medical University Nanfang Hospital

Zhu Zhang

Southern Medical University Nanfang Hospital

Daogang Guan

Southern Medical University

Guanglong Huang ( $\sim$ hgl1020@163.com )

Southern Medical University Nanfang Hospital https://orcid.org/0000-0002-1589-9202

\section{Research}

Keywords: Xuefu Zhuyu Decoction, glioblastoma, network pharmacology, key functional network, cumulative contribution rate

Posted Date: January 11th, 2021

DOI: https://doi.org/10.21203/rs.3.rs-141955/v1

License: (c) (i) This work is licensed under a Creative Commons Attribution 4.0 International License. Read Full License 


\section{Abstract}

Background

Xuefu Zhuyu Decoction (XFZYD) is a well-known Traditional Chinese Medicine (TCM) formula that has many pharmacological effects, including enhancing immune function, improving hemorheology and regulating blood vessels bidirectionally. Modern pharmacological and clinical studies showed that XFZYD could ameliorate curative effect of glioblastoma (GBM). The aim of this study was to interpret core components and the hidden molecular mechanisms of XFZYD on GBM.

Methods

Here, a novel network pharmacology strategy, which combined pharmacological data, next generation sequencing data, pharmacokinetic parameters and a novel node importance calculation method was designed to decipher the potential therapeutic mechanism of XFZYD on GBM. The partial components in core component group (CCG) were evaluated by in vitro expriments. We identified 117 chemical components analysis through ADME screening, then componenttarget network and GBM related genes were integrated as the component-target-pathogenic gene (C-T-P) network.

Results

The results show that the enriched pathways of targets in the key functional network could cover $77.92 \%$ of the enriched pathways of pathogenic genes. A novel cumulative contribution rate (CCR) calculation model was designed and captured CCG with 21 components. The statistics results indicate that 15 enriched pathways of the targets of CCG were overlap with pathogenic genes enriched pathways. Finally, some core components in CCG were validated by in vitro experiments.

Conclusion

The results show that our proposed stategy for decoding CCG and infering the underlying mechanism with good reliability and accuracy. The validation results indicate that the CCG play a therapeutic role on GBM by targeting to PI3K-Akt signaling pathway and Toll-like receptor signaling pathway. Our strategy provides methodological reference for the optimization and secondary development of TCM formula.

\section{Background}

Glioma is the most common primary tumor of the central nervous system (CNS), accounting for more than $40 \%$ of intracranial tumors [1]. According to the classification of the World Health Organization, gliomas can be classified into I-IV grade, in which IV glioma, also known as glioblastoma (GBM), accounting for more than $50 \%$ of all gliomas (David N. Louis and Dominique Figarella-Branger, 2016). After receiving standard STUPP regimen [2, 3], the median survival time of GBM patients was still only 12.1 to 14.6 months, and only $3 \%$ to $5 \%$ of the patients had a median survival time of more than 3 years [1]. Therefore, it is urgent to seek alternative treatment methods in treating GBM. Increasing evidence confirms that some formulas and components in traditional Chinese medicine (TCM) have the anti-glioma effect, such as Xuefu Zhuyu Decoction (XFZYD) can inhibit the invasive spread of glioma stem cells [4], Oxymatrine, an alkaloid derived from Sophora flavescens Aiton usually used to inhibit GBM cells proliferation and induce apoptosis [5], Aloperine, an alkaloid extracted from the leaves of Sophora alopecuroides, acts as a underlying inhibitor of anti-apoptotic Bcl-2 protein in glioma cells [6], Hedyotis diffusa Willd extract could inhibits human glioblastoma cell growth through the AKT / ERK pathway [7, 8], and chloroform extract from Angelica sinensis (AS-C) regulated GBM cells cycle arrest and apoptosis to play an anti-tumor role [9].

Among these formulas and components, XFZYD has the effects of promoting blood circulation and removing blood stasis. It is commonly used in the clinical treatment of brain injury [10], neurological dysfunction [11], coronary heart disease [12], and cognition impairment [13]. XFZYD is prepared from 11 kinds of herbs, which include Semen Persicae (SP, the dried mature seed of Prunus persica (L.) Batsch)(12g), Carthamus tinctorius L. (9g), Radix Angelicae Sinensis (RAS, the dried root of Angelica sinensis (Oliv.) Diels)(9g), Radix Rehmanniae (RR, the dried root tuber of Rehmannia glutinosa (Gaertn.) DC.)(9g), Radix Achyranthis Bidentatae (RAB, the dried root of Achyranthes bidentata Blume)(9g), Rhizoma Chuanxiong (RC, the dried rhizome of Ligusticum chuanxiong Hort.)(4.5g), Radix PlatycodonisL (RP, the dried root of Platycodon grandiflorus (Jacq.) A.DC.)(4.5g), Radix Paeogiae Rubra (RPR, the dried root of Paeonia lactiflora Pall.)(6g), Fructus Aurantii (FA, the dried immature fruit of Citrus aurantium L.)(6g), Radix Glycyrrhizae (RG, the dried root of Glycyrrhiza uralensis Fisch. ex DC.)(6g), Radix Bupleuri (RB, the dried root of Bupleurum chinense DC.) $(3 \mathrm{~g})$. Some components and herbs in this formula have been reported in the literature for GBM treatment, tetramethylpyrazine (TMP) extracted from the TCM herb Rhizoma Chuanxiong, had potential curative effect on gliomas, via the inhibition of cell proliferation [14]. Danggui may be used to treat high-grade astrocytomas, by inhibiting the VEGF expression to inhibit tumor growth [15] The formula also been reported has an anti-glioma effect, Guangmei Lu et al. showed that the treatment of 3 cases of glioma with XFZYD prolonged progressionfree survival (PFS) of the patients (Ronghui, 2017). Jianmin Liu et al. showed that XFZYD inhibits the invasive spread of glioma stem cells [4]. But, the specific mechanism of XFZYD in treating GBM is not clear.

Increasing evidence shows that network pharmacology plays an critical role in detecting the pharmacological effects of natural products on a variety of complex diseases. Network pharmacology is based on multi-level and multi-angle interactive networks, which represent the interaction of component target, pathogenic gene, pathway and disease. It can systematically and comprehensively observe the intervention and influence of drugs on disease networks, thus revealing the synergistic mechanism of key components in drugs on the complex disease. Network pharmacology is usually used to analyze the mechanism of drug action, find lead compounds or new indications, and determine new drug targets from the system level. The overall and systematic characteristics of network pharmacology are not only consistent with the concept of diagnosis and treatment of diseases by TCM, but also consistent with the cooperative action principle of TCM formula. 
At present, a novel systematic pharmacological model was designed to detect the core component group (CCG) and explore the therapeutic mechanism of XFZYD in treating GBM. Our proposed strategy could combine pharmacokinetics synthesis screening data, high throughput data, target fishing data, network analysis, and molecular docking to detect CCG and infer the potential mechanisms of XFZYD in treating GBM. Specifically, the chemical components of XFZYD are collected in public databases. Then we use the previously proposed ADME model to screen potential active components of XFZYD, and then predicted targets of these components to construct a C-T network. After that, we integrate the C-T network and pathogenic genes into a comprehensive C-T-P network and then use the node importance calculation method to obtain the key functional network. Based on the key functional network, we use cumulative contribution rate (CCR) to select the CCG that can target most of the genes in the key functional network and speculate the molecular mechanism of XFZYD in treating GBM on CCG.

\section{Methods}

\section{Construct Weighted GBM-related Pathogenetic Gene network}

Two datasets, GSE50161 and GSE15824 in GEO database [16] recorded the expression profile data of normal and GBM patients were kept for futher analysis.. We use "limma" package to obtain the differential expression genes $(p<0.05$ and fold change $>2)$ of the two datasets, respectively. the shared part of these two differential expression genes is considered to be reliable GBM pathogenic genes. Each pathogenic gene has two fold change values, Then we used the square root of the two fold change value as the weight of pathogenic gene.

PPI data collected from the public databases STRING, Dip, BioGRID, Mint, and HPRD [17] were combined as a comprehensive PPI network. The GBM-related pathogenetic genes with defined weight were mapped to the comprehensive PPI network to construct the weighted GBM-related pathogenetic gene network.

\section{Component Identification}

TCMSP could provide detail items for each component, mainly include drug-likeness (DL), oral bioavailability, (OB), blood-brain barrier (BBB), and so on. Shanghai Institute of Organic Chemistry, Chinese Academy of Sciences. Chemical database [DB/OL] can provide information on the structure and identification of compounds, natural products and pharmaceutical chemistry, safety and environmental protection, chemical literature, chemical reactions and comprehensive information. TCMID contains about 47000 prescriptions, 8159 herbs, 25210 compounds, 6828 medicines, 3791 diseases and 17521 related targets, which is the most integrated collection of big data in the related field of TCM research. TCM @ Taiwan contains 443 kinds of TCM formula and more than 20000 pure ingredients, which are divided into different categories and provide enquiries, including inquiries about the ingredients of TCM. All the ingredients of XFZYD come from the above databases. Open Babel Toolkit (version 2.4.1) was uesd to prepare all chemical structures and converte to canonical SMILES. HitPick [18], Similarity Ensemble Approach (SEA ) [19], STITCH [20] and Swiss Target Prediction [21] were used to predict the targets of XFZYD.

\section{ADME Screening}

The preliminary evaluauion of ADMET properties is becoming an important process in modern drug discovery. In our study, three ADMET-related models of DL, $\mathrm{OB}$, and BBB were used to select the active components in XFZYD. Anatomically, BBB is characterized by the continuous tight junctions between continuous non-fenestrated endothelial cells. The function of this connection is usually to restrict the entry of protein and underlying drugs into the brain parenchyma (Tattersall et al., 1975). It is important to assess the ability of each component to enter the CNS, and components with >- 0.3 are considered as candidate components. $\mathrm{OB}(\% \mathrm{~F})$ represents the percentage of the drug reaching the systemic circulation with unchange in oral administration, that indicates the trends of ADMET process (Xu et al., 2012). Higher OB usually represent the core index to define the characteristics of active components as potential drugs. The compounds with $\mathrm{OB} \geq 30 \%$ were selected as candidate drugs. Drug-likeness (DL) is a digital indicator that is widely used in drug design to assess the drug likeness of an expected components, which helps to capture pharmacokinetics and drug characteristics. The DL value is more than 0.14 can be used as the selection standard for the TCM components [19].

\section{Construct C-T network}

The Cytoscape (version 3.5.1) [22] was employed to constructed and visualized the component-target (C-T) network of XFZYD. The Cytoscape plugin NetworkAnalyzer [23] was uesd to calculate topological parameters of networks.

\section{Develop a PageRank algorithm model to Select key functional network}

In tumor research, the PageRank has employed to analysis the important nodes in the protein network [24, 25]. In order to find key functional network of XFZYD in treating GBM, the following mathematical algorithm was designed and described:

Here, the PageRank formula was revised by adding damping factor $d$ to the simple formula:

$$
\operatorname{PR}(\mathrm{A})=(1-\mathrm{d})+\mathrm{d} * \sum_{i=1}^{m} \frac{P R(T i)}{C(T i)}
$$

$\mathrm{PR}(\mathrm{A})$ represent the importance of the node $\mathrm{A}$. $\mathrm{Ti}$ is a component or target linking to $\mathrm{A} . \mathrm{C}(\mathrm{Ti})$ is the number of links of Ti. $\mathrm{D}$ is the damping coefficient.

\section{Cumulative Contribution Rate (CCR) Calculation}


In order to get the core component group (CCG), the CCR model was designed to extract CCG from the key functional network, which would be used to infer the hidden mechanism of XFZYD in the therapy of GBM. Given $n$ components and cumulative contribution rates, the number of pathways enriched in component $i$ is $W_{i}$, the coverage rate of the enriched pathway in components compare to the pathogenic gene is $P_{i}$ and the total restriction condition $C$ is the number of components was used to calculate the CCR. Select the appropriate components to calculate the contribution rate based on coverage of enriched pathways in components compare to that in pathogenic genes. When the CCR reaches or exceeds $90 \%$, the iterative cumulative calculation of this model will be terminated. There are only two options for each component $i$ : included or not, that is, component $i$ can only be included in the cumulative probability once. The model of this problem can be expressed as the following $0 / 1$ integer programming model:

Subject to $\bigotimes$

$$
\begin{aligned}
& \max f\left(x_{1}, x_{2} \cdots, x_{n}\right)=\sum_{i=1}^{n} c_{i} x_{i} \\
& \text { s.t }\left\{\begin{array}{c}
\sum_{\substack{i=1 \\
x_{i} \in\{0,1\}}}^{n} w_{i} x_{i} \leq p_{i} \\
(i=1,2, \cdots n)
\end{array}\right.
\end{aligned}
$$

Where $x_{i}$ is the decision variable of $0-1, x_{i}=1$ represents the item is chosen into the knapsack, and $x_{i}=0$ represents the item is not selected into the knapsack.

\section{Gene Ontology and Pathway Analysis}

The R package clusterProfiler (Yu et al., 2012) was used to perform gene ontology (GO) analysis and pathway enrichment analyses [26]. In enrichment analysis, $\mathrm{P}$ value less than 0.05 is considered to be significant, and these enriched images are displayed by ggplot2 in R. Different colors in the pathway diagram are marked by Pathview package in R Bioconductor (Luo et al., 2017).

\section{Experimental Validation}

\section{Chemicals and Reagents}

Fetal bovine serum (FBS, Gemini Foundation) and Dulbecco's modified Eagle's medium (DMEM, Biological Industries) were purchased from Gibco, $\beta$-actin (Cell signaling technology, 1:2000), Bcl-2 (Cell signaling technology, 1:1000).

\section{Cell Culture}

The human LN229 glioma cells (the American Type Culture Collection) were cultured inwith complete medium in an incubator with $37{ }^{\circ} \mathrm{C}$. When LN229 cells reached $80-90 \%$ confluency, they were treated with different concentrations of baicalein and wogonin for $24 \mathrm{~h}$, respectively.

\section{Cell Viability Assay}

CCK8 assay was utilized to measure cell viability. Cells were seeded in 96-well plates. Following incubation for $24 \mathrm{~h}$, the original medium was replaced with medium containing different concentrations of baicalein and wogonin (Jingzhu Biotechnology Co., Ltd, Nanjing, China) for $24 \mathrm{~h}$. Subsequently, $10 \mu \mathrm{L}$ of CCK8 (KeyGEN BioTECH, KGA317) reagent was added to each well and the 96-well plates were incubated for $2 \mathrm{~h}$ in the dark. Finally, the optical density (OD) values were measured at $450 \mathrm{~nm}$ using a microplate reader.

\section{Western blot analysis}

The LN229 cell was lysed by RIPA Buffer (Solarbio) at $4{ }^{\circ} \mathrm{C}$ for $30 \mathrm{~min}$ to obtain protein lysates. $30 \mathrm{mg}$ proteins was separated using a $10 \%$ SDS-PAGE and transferred onto PVDF membranes (Invitrogen, Carlsbad, CA). The antibodies were used in this analysis. chemiluminescence signals were detected with ECL reagent (Millipore, USA).

\section{Measurement and detection of cell apoptosis}

Cell apoptosis was assessed using FITC Annexin V Apoptosis Detection Kit (KeyGEN BioTECH) according to manufacturer's instructions. Cells were treated with $60 \mu \mathrm{M}$ baicalein and $80 \mu \mathrm{M}$ wogonin for $24 \mathrm{~h}$. We washed with PBS and collected cells. The cells were suspended in annexin V binding buffer. Finally, $2 \mu \mathrm{l}$ of annexin V-FITC and $2 \mu$ of propidium lodide (PI) were utilized to incubated in dark conditions for $5 \mathrm{~min}$.

\section{Statistical Analysis}

In order to compare the molecular characteristics of each component in XFZYD, statistical analysis was performed using SPSS22.0.The student's t-test for comparison was used to analysis data.

\section{Results}

In our study, we designed a systematic pharmacological method to analyze the molecular mechanism of XFZYD in treating GBM (Figure 1). The key point of the method is to construct the weighted pathogenetic gene network and C-T network, then integrate the two networks through PPI data to construct a 
comprehensive C-T-P network, and then optimize the C-T-P network by using the novel node importance method to capture the key functional network. The dynamic programming algorithm was employed to predict CCG, finally, the CCG and their targets were used to infer the molecular mechanism of XFZYD in treating GBM. This strategy provided a methodological reference for formula optimization in TCM.

\section{Construct Weighted Pathogenetic Gene network}

The weighted pathogenetic gene network reflects the pathogenesis of glioma assisted by multiple genes. thus, the construction of pathogenic gene regulatory network is the key to provide evidence for glioma therapy. In this study, we use the pathogenetic genes (Figure S1) and comprehensive PPI network (Figure S2) to construct the weighted pathogenetic network. In this network, the highest weight gene is RRM2, following by TOP2A, MELK, NUSAP1, NDC80, DLGAP5, CHI3L1, IGF2BP3, METTL7B, and COL3A1. Most of these pathogenic genes were reported associated with cell apoptosis related pathways of GBM (Figure S3). Such as, Rikke et al. find that regulated RRM2 expression promoted tumorigenicity of GBM [27]. Arivazhagan et al found that the expression of TOP2A was a prognostic biomarker for GBM patients treated with TMZ [28]. Marie et al. confirmed that there exists a significant correlation between the expression of MELK and malignant grade of astrocytoma [29]. Qian et al. demonstrated that NUSAP1 could be used as a new prognostic indicator and a underlying target for GBM [30]. Clinical trials could be conducted to verify the efficacy of gene therapy by the down-regulating BUB1B (or NDC80) in GBM patients [31]. These results show that the weighted pathogenetic genes network and weighted pathogenetic genes can reflect the pathogenesis of GBM, and also provide a reliable reference for the next step of constructing an intervention network (Figure S4).

\section{Chemical components analysis}

Chemical analysis is one of the main means to study the material basis and action mechanism of herbal medicine. Through literature search, we collected the chemical constituents and concentrations of various components in XFZYD. The detailed information was shown in Table 1. The results show that the chemical components of some herbs have high concentrations, suggesting that these components may have therapeutic effects and provide an extended experiment-aided chemical space for screening of active components and providing a more comprehensive reference for further analysis [3, 32, 33].

Among these document-confirmed components of XFZYD, paeoniforin [34-36], Gallic acid [37-39], Protocatechuic acid [40] and Chlorogenic acid [41-43] with more evidences that have an effect on GBM.

\section{Active components of XFZYD}

To fully understand the potential anti-GBM mechanism of XFZYD, we collected components of the 11 herbs in XFZYD from published databases, a total of 1365 components were collected. To assess the pharmacokinetic properties of the XFZYD formula, we used published ADME system to predict the pharmacological properties of BBB, OB, and DL. Only components with BBB $\triangle-0.3,0 B \otimes 30 \%$, and DL $\triangle 0.14$ were predicted as active components. After screening, 117 active components were kept for further analysis. The detailed information of the active components can be found in Table 2 .

\section{C-T network construction}

In order to observe the relation pattern of active components and their corresponding targets in XFZYD, Cytoscape was empolyed to construct C-T networks (Figure 2). The C-T network consists of 117 active components targeting to 855 genes through 4488 edges. Then the plug-in of Cytoscape, NetworkAnalyzer was used to analyze the topology parameters of th C-T network and showed that the average degree of targets and components in XFZYD were 5.25 and 39.40 , respectively. The average number of components for per target is 5.25. This result indicates that XFZYD has multi-components feature in treating GBM Among these components, gadelaidic acid (MOL004996, degree=123) has the highest number of targets, followed by icos-5-enoic acid (MOL004985, degree=115), cacetin (MOL001689, degree=107), 7-Methoxy-2-methyl isoflavone (MOL003896, degree=105), wogonin (MOL000173, degree=96), Glypallichalcone (MOL004835, degree=96), 1,3-dihydroxy-9-methoxy-6-benzofurano[3,2-c]chromenone (MOL004913, degree=90), Jaranol (MOL000239, degree=90), Mandenol (MOL001494, degree=86), and linolenic Acid (MOL000432, degree=84). Most of these components were reported associated with cell apoptosis related pathways of GBM. Such as cacetin (MOL001689, degree=107) showed lower IC50 values and presenting promising antit-glioma effects (Teles et al., 2015), wogonin (MOL000173, degree=96) induced human glioma cell apoptosis mediated reactive oxygen species (ROS) generation (Tsai et al., 2012), linolenic Acid (MOL000432, degree=84) was a selective activity against GBM cells compare normal brain(Das, 2007).

In the C-T network, the average number of targets for each component is 38.40. This result indicates that XFZYD has a multi-targets feature in treating GBM. In the top of 30 targets with larger weight include ESR2, ESR1, ABCG2, CYP19A1, PTN1, CA12, etc. Interestingly, the majority of these targets are related to pathogenesis or treatment of GBM. For example, ESR1 (degree=93) has GBM suppressor function and ER $\beta$ isoform switching induces GBM progression (Liu et al., 2018), ABCG2 (degree=81)

was an important indicator of patient prognosis, and ABCG2 inhibitor may be a potential treatment strategy for GBM (Emery et al., 2017)(Liu and Sareddy et al., 2018), the CA12 (degree=26) inhibitor prolongs the survival of TMZ-treated GBM-bearing mice [44]. Thus, these components could be considered as curative elements in treating GBM.

\section{Key Functional Network Identification}

The multi-component and multi-target characteristics of TCM determine that it exists network intervention in the process of treating complex diseases, and the active components in the formula can transmit the intervention effect to pathogenic genes through PPI networks by influencing their targets, thus playing a therapeutic role on diseases. Components-targets-pathogenic genes form a complex intervention network, in which some components play a crucial role and some components play an auxiliary role. How to find the core component groups and key functional networks from this network is the foundation to understand the treatment of complex diseases of formula in TCM and is also the foundation for the secondary development of TCM formula. PageRank 
(PRK) algorithm usually used in the Google search engine to measure the importance of web pages, is also widely used in biomedical researches. Syed et al. used PRK centrality to consistently find convincing eloquent brain areas (Perry et al., 2019). Hao Zhang et al. predicted human and plant target genes using RNAhybrid by PRK algorithm (Zhang et al., 2016). In this paper, we combined C-T network, weighted pathogenetic gene network and PPI network to form a comprehensive network, in this network, we use PRK value to describe the importance and influence of nodes, the nodes with higher PRK value than the average and median PRK value were defined as A functional network and M functional network, respectively. In the further analysis of A functional network and $\mathrm{M}$ functional network, we found that $\mathrm{A}$ functional network includes 37 components and 728 targets, $\mathrm{M}$ functional network includes 33 components and 688 targets. Then we performed pathway analysis by using targets of A functional network, M functional network and C-T network respectively. Among them, the number of targets in A functional network enriched pathways was $131(\mathrm{p}<0.05)$, the number of targets in M functional network enriched pathways was $125(p<0.05)$, the number of genes in C-T network enriched pathways was $154(p<0.05)$. Compared with enriched pathways of genes in the C-T network, the coverage rates of the two strategies were $77.92 \%$ and $74.68 \%$, respectively (Figure 3 ). According to this result, we used the A functional network as key functional network for further analysis.

\section{CCG Prediction and validation}

\section{CCG Prediction}

The key functional network contains a large number of chemical components, action targets and interactions between targets, which form a complex response network. There are potentially effective component groups and modes of action in this network. How to detect these effective component groups and action patterns is key to understand the treatment of glioma with XFZYD. Here we design CCR model to obtain CCG by heuristic optimization of key functional networks using reasonable global metrics. According to the results of CCR model, the top 5 components including gadelaidic acid (MOL004996), icos-5-enoic acid (MOL004985), acacetin (MOL001689), 7-Methoxy-2-methyl isoflavone (MOL003896), and Glypallichalcone (MOL004835) contribute to $55.63 \%$ target coverage of genes in key functional network. For further analysis, 21 components can contribute to $90.66 \%$ targets coverage of genes in key functional network were selected as CCG (Figure 4 and Table 3). Higher targets coverage of genes in key functional network proved that the CCG may play a leading role and produced combination actions in treating GBM.

\section{CCG Validation}

To analysis of XFZYD in the treatment of GBM at the functional level, we used CCG targets for pathway analysis, the number of CCG targets enriched pathways was $121(p<0.05)$, and the number of pathogenic genes enriched pathways was $26(p<0.05)$. We defined the shared enrichment pathways of pathogenic genes and targets in key functional network as the reference pathways. The CCG targets enriched pathways account for $78.95 \%$ of the reference pathways (Figure 5A). This results confirmed the accuracy and reliable of our CCG selection model. For futher analysis, we found that these main targets of CCG were frequently involved in PI3K/Akt signaling pathway (hsa04151), Toll-like receptor signaling pathway (hsa04620), Proteoglycans in cancer (hsa05205), Cell cycle (hsa04110), Cellular senescence (hsa04218), and Platelet activation (hsa04611), etc (Figure 5B). For example, PI3K/AKT pathway plays a key role in the occurrence and development of GBM [45]. AKT, as a regulatory molecule and a potential drug target, has been widely studied for its carcinogenicity [46, 47]. AKT activation promotes tumor progression by affecting tumor proliferation and growth [48]. Up to now, three isomers of AKT (AKT1, AKT2 and AKT3) have been discovered. According to reports, AKT3 can significantly activate DNA repair to resist radiotherapy and chemotherapy in GBM patients (Turner et al. (2015). Results show that our combined optimized strategy of key functional network detection and CCR model is accurate and reliable, and our predicted core component group plays an critical role in treating GBM.

\section{GO enrichment analysis of CCG targets}

To further analyze the combined effects of XFZYD, all targets of CCG were enriched by GO enrichment analysis (Figure 6B). We defined the shared enriched GO terms of key functional network and pathogenetic gene as the therapeutic response GO terms. The enriched GO terms of CCG targets account for $73.68 \%$ of the therapeutic response $\mathrm{GO}$ terms (Figure 6A).

GO enrichment analysis indicated that the targets regulated by CCG of XFZYD were mainly enriched in oxidative stress and lipid metabolism. For example, the GO terms of oxidative are response to oxidative stress (G0:0006979, CYP1B1, LCN2, PTGS1, FABP1, PTGS2, CDK1, etc.), decreased oxygen levels (G0:0036293, CYP1A1, PRKCE, RORA, ADORA1, PPARA, PPARD, TERT, etc.), hypoxia (G0:0001666, CYP1A1, PRKCE, RORA, ADORA1, PPARA, PPARD, TERT, etc.), and cellular response to oxidative stress (G0:0034599, CYP1B1, LCN2, FABP1, CDK1, MCL1, NOX4, MMP2, etc.). Important genes involved in oxidative stress in these GO terms include PPARG, PPARA, PPARD, TERT, CDK1, etc. These genes are involved in the oxidative stress of GBM by fostering proliferation and affecting survival and migration [49-51]. The GO terms of lipid metabolism are regulation of lipid metabolic process (GO:0019216, CYP1A1, NCOA1, PRKCE, FABP3, NR1H3, RORC, HMGCR, CYP51A1, etc.), lipid transport (G0:0006869, ABCB1, CYP19A1, ABCC1, NCOA1, SLCO2B1, PLA2G2E, FABP4, FABP3, etc.), lipid localization (G0:0010876, ABCB1, CYP19A1, ABCC1, NCOA1, SLCO2B1, PLA2G2, etc.), response to lipopolysaccharide (G0:0032496, CYP1A1, PRKCA, LCN2, MAOB, PRKCE, NR1H3, PPARD, etc.) and lipid catabolic process (G0:0016042, FABP7, CYP1B1, CYP19A1, FAAH, PLA2G2E, SRD5A1, PRKCE, etc.). Important genes involved in lipid metabolism in these $\mathrm{GO}$ terms include ABCB1, ABCC1, FABP4. These genes are involved in the lipid metabolism of GBM by influencing cell growth, proliferation, angiogenesis and invasion [52,53]. GO analysis confirmed that XFZYD treats GBM by regulating of oxidative stress and lipid metabolism.

\section{Pathway analysis to explore the potential therapeutic mechanisms of XFZYD}

In order to investigate the potential therapeutic mechanisms of XFZYD on GBM, We analyzed the intersection of enrichment pathways of CCG target gene and GBM pathogenic genes, totally get 15 shared pathways. Among the 15 common pathways, remove the pathways associated with other diseases, the PI3K-Akt 
signaling pathway (hsa04151) and Toll-like receptor signaling pathway (hsa04620) were the literature proved GBM-related pathways. For example, PI3K-Akt signaling pathway plays an important role in cell cycle, apoptosis, and cell proliferation in GBM [54, 55].

In order to analysis the synergetic mechanism of XFZYD in treating of GBM, we constructed a comprehensive signaling pathway by combining two principal molecular pathways. As shown in Figure 7, we defined the first three columns as the upstream position of the pathway and the remaining columns as the pathway downstream position. Among them, targeting to the PI3K-Akt signaling pathway is the main approach for XFZYT to treat GBM. XFZYD regulates 7 targets located the upstream, such as Syk, RTK, TLR2 / 4, and 32 targets located PI3K-Akt signaling pathway downstream, such as MEK, AKT, and mTOR. Downstream targets account for $82.05 \%$. XFZYD may activate downstream of AKT proteins through upstream RTK, leading to cascade amplification of downstream GSK3 and CREB, which was closely related to GBM cell proliferation and protein synthesis [48].

Toll-like receptor signaling pathway is also a critical way for XFZYD to treat GBM. The target of XFZYD regulation is located the pathway downstream. Such as, 11 targets of XFZYD, including AKT are located downstream of the Toll-like receptor signaling pathway. XFZYD can affect upstream TLR2/4 and Rac1 and then activate downstream AKT, thereby further affecting a series of malignant biology with GBM behavior-related proteins, such as STAT1, NFKB, and IL-6, thus play an important role in the treatment of GBM.

\section{Experimental Validation in Vitro}

The effects of baicalein and wogonin in CCG with different concentrations on the viabilities of human LN229 glioma cells were detected by CCK8 assay. After $72 \mathrm{~h}$ of incubation, the cell viabilities of LN229 cells were $79.63 \%, 67.68 \%, 50.38 \%, 44.61 \%, 36.28 \%$ and $88.61 \%, 78.91 \%, 66.88 \%, 53.36 \%, 44.53 \%$ after exposure to baicalein and wogonin at concentrations of $40,60,80$, and $100 \mu \mathrm{M}$, respectively (Figure $8 \mathrm{~A}$ ). The above results demonstrated that baicalein and wogonin remarkably suppressed cell proliferation in LN229 cells.

According to the results of cell proliferation effect of baicalein and wogonin, we found $60 \mu \mathrm{M}$ baicalein and $80 \mu \mathrm{M}$ wogonin could markedly inhibit the proliferation of LN229 cells. Therefore, $60 \mu \mathrm{M}$ baicalein and $80 \mu \mathrm{M}$ wogonin were used to evaluate the effect on apoptosis in LN229 cells for $24 \mathrm{~h}$. Compared with the control group, Necrosis and late apoptotic cells of upper right quadrant was increasing ( $65.1 \%$ vs $0.59 \%, 52.2 \%$ vs $0.59 \%$, respectively) with baicalein and wogonin treatment (Figure 8B). Further, we found that treatment with baicalein and wogonin could significantly reduced Bcl-2 protein expression (Figure $8 \mathrm{C})$. Our data suggested that the baicalein and wogonin induced apoptosis phenomenon play a important role in inhibiting the development of GBM.

\section{Discussion}

For thousands of years, TCM adopts the method of holistic treatment, emphasizing the holistic view, systematic view and cooperative view in the application of formulas in treating complex diseases. Network pharmacology has distinguishing feature of systematicness and integrity, which accord with the theoretical basis of TCM. Network pharmacology emphasizes the regulation of multi-component and multi-target of signal pathways to improve the efficacy of drugs and reduce toxic and side effects. Now, network pharmacology is widely applied to study the complex diseases treatment in TCM.

More and more experiments show that some components in TCM formula have synergistic and antagonistic effects, and even some components may produce toxic and side effects. How to use the current computing technology and chemoinformatics technology to detect the core components and analyze the mechanism of TCM formula are a major challenge of network pharmacology. The main purpose of optimization of TCM formula is to decrease unnecessary functional components and improve the therapeutic effect of the formula. Through the optimization of the TCM formula, medicinal materials or components with certain drug effects can be screened, thus the formula is more clarified, and the therapeutic effect is more enhanced. In order to better optimize the TCM formula with clinical effect and obtain the main components and key mechanisms, the network pharmacological model and mathematical model are adopted to obtain the optimal target group by using the change of the proportion of the target enrichment pathway to the pathogenic gene enrichment pathway. Based on these target groups, the optimal key component group is speculated, and the function evaluation and mechanism speculation are carried out. It provides a methodological reference for the optimization and secondary development of TCM formula.

The emergence of accumulated high-throughput data provides rich data support for the characterization of human diseases, such as 1) looking for application of blood-based biomarkers in early diagnosis of tumors [31]. 2) integrate epidemiological, pharmacological, genetic and intestinal microbiome data into the drug metabolite map [56]. 3) the next generation tool for rapid disease screening [57]. These data make it possible to interpret the therapeutic mechanism of TCM in the treatment of complex diseases based on network pharmacology and high-throughput sequencing data. How to use this strategy is still in its infancy.

In this study, the mathematical methods and network pharmacology were used to study the optimization strategy of component prescription, functional analysis of target genes of key component groups after optimization and changes in the coverage rate of related pathogenic gene enrichment pathways, so as to better optimize the clinical effect of classical prescriptions. The results show that the enrichment pathway of the key functional network can cover $77.92 \%$ of the gene enriched pathways of C-T network, further confirming that our strategy of constructing a key functional network and selecting effective proteins is reasonable and reliable. Based on the effective protein provided by the key functional network, the cumulative contribution rate is calculated by CCR model, and finally the target coverage rate was calculated, CCG with 21 active components reaches more than $90 \%$ target coverage rate. Bioinformatics analysis demonstrated that our optimized CCG was closely related to the pathogenesis of GBM at functional level. Cellular experiments verified the reliability of the network pharmacology strategy by verifying the inhibitory effects of key components in CCG on LN29 cells. This proves once again the reliability and accuracy of our key functional network and CCR model.

\section{Conclusions}


At present, our network pharmacology model provides a powerful tool for optimizing the core components group and decoding the underlying mechanism of TCM formula. There are still some limitations. For example, in animal medicine research of XFZYD, how to select less active ingredients to verify the therapeutic effect and molecular mechanism of them in treating GBM by vivo experiments.

\section{Abbreviations}

GBM (glioblastoma ); TMZ (temozolomide); XFZYD (Xuefu Zhuyu Decoction); C-T-P network (component-target-pathogenic gene network); CCR (cumulative contribution rate); CCR (core component group); TCM (traditional Chinese medicine); KEGG (Kyoto Encyclopedia of Genes and Genomes); GO (Gene Ontology); CNS (Central Nervous System); DL (drug-likeness); OB (oral bioavailability); BBB (blood-brain barrier).

\section{Declarations}

\section{Competing interests}

The authors declare that they have no competing interests.

\section{Author's contributions}

${ }^{*} \mathrm{ZYL}$ and $\mathrm{KXW}$ contributed equally to this work.

GLH and DGG provided conception and design of the work. ZYL, KXW, RWY, GZY, ZYL, YWL, DGG, and GLH conducted acquisition, analysis and interpretation of data. ZYL, KXW, DGG, and GLH contributed to critical revision for important intellectual content.

All Authors read and approved the manuscript.

\section{Acknowledgements}

Not available.

\section{Funding}

This work was partially supported by the Startup fund from Southern Medical University [grant No. G820282016], the Guangdong Science and Technology Department (2016A040403053), the Natural Science Foundation Council of China [grant No. 31501080, 32070676], Hong Kong Baptist University Interdisciplinary Research Matching Scheme [grant No. RC/IRCs/17-18/04] and Outstanding Youth Development Scheme of Nanfang Hospital, Southern Medical University (2019J002). The funder had no involvement in the study.

\section{Availability of data and material}

The data analyzed in this study can be accessed by sending a request to the corresponding author.

\section{Ethics approval and consent to participate}

A written consent was obtained from a patient. The Institutional Review Board of Nanfang Hospital affiliated to Southern Medical University approved the study.

\section{Consent for publication}

Written informed consent was obtained from the patients for publication of this case reports. All the authors listed have approved the enclosed manuscript.

\section{References}

1. Louis DN, Perry A, Reifenberger G, von Deimling A, Figarella-Branger D, Cavenee WK, Ohgaki H, Wiestler OD, Kleihues P, Ellison DW: The 2016 World Health Organization Classification of Tumors of the Central Nervous System: a summary. Acta Neuropathol 2016, 131(6):803-820.

2. Karachi A, Dastmalchi F, Mitchell DA, Rahman M: Temozolomide for immunomodulation in the treatment of glioblastoma. Neuro Oncol 2018, 20(12):1566-1572.

3. Yan-Hua Z, Yu-Ping T, An-Wei D, Li Z, Sheng G, Jin-Ao DJCJoPA: HPLC determination of six aromatic acids in four traditional Chinese medicine formulae for promoting blood circulation to remove stasis. Chinese Journal of Pharmaceutical Analysis 2010.

4. Jianmin L, Liangwen H, Peng HUJJoNCM: Influence of Blood-activating and Stasis-eliminating Chinese Herbal Medicine on Invasive Behavior and TIMP1 mRNA Expression in C6 Brain Tumor Stem Cells. Journal of New Chinese Medicine 2013.

5. Liu F, Wang B, Wang J, Ling X, Li Q, Meng W, Ma J: Oxymatrine Inhibits Proliferation and Migration While Inducing Apoptosis in Human Glioblastoma Cells. Biomed Res Int 2016, 2016:1784161.

6. Xu Z, Wang X, Chen X, Zeng S, Qian L, Wei J, Gong Z, Yan Y: Identification of Aloperine as an anti-apoptotic Bcl2 protein inhibitor in glioma cells. PeerJ 2019, 7:e7652.

7. Chen X, Li XF, Chen Y, Zhu S, Li HD, Chen SY, Wang JN, Pan XY, Bu FT, Huang C et al: Hesperetin derivative attenuates CCI4-induced hepatic fibrosis and inflammation by Gli-1-dependent mechanisms. Int Immunopharmacol 2019, 76:105838. 
8. Zhang Y, Xie RF, Xiao QG, Li R, Shen XL, Zhu XG: Hedyotis diffusa Willd extract inhibits the growth of human glioblastoma cells by inducing mitochondrial apoptosis via AKT/ERK pathways. J Ethnopharmacol 2014, 158 Pt A:404-411.

9. Tsai NM, Lin SZ, Lee CC, Chen SP, Su HC, Chang WL, Harn HJ: The antitumor effects of Angelica sinensis on malignant brain tumors in vitro and in vivo. Clin Cancer Res 2005, 11(9):3475-3484.

10. Fu C, Wu Q, Zhang Z, Xia Z, Ji H, Lu H, Wang Y: UPLC-ESI-IT-TOF-MS metabolomic study of the therapeutic effect of Xuefu Zhuyu decoction on rats with traumatic brain injury. J Ethnopharmacol 2019, 245:112149.

11. Zhu L, Tang T, Fan R, Luo JK, Cui HJ, Zhang CH, Peng WJ, Sun P, Xiong XG, Wang Y: Xuefu Zhuyu decoction improves neurological dysfunction by increasing synapsin expression after traumatic brain injury. Neural Regen Res 2018, 13(8):1417-1424.

12. Tao T, He T, Wang X, Liu X: Metabolic Profiling Analysis of Patients With Coronary Heart Disease Undergoing Xuefu Zhuyu Decoction Treatment. Front Pharmacol 2019, 10:985.

13. Yeh CW, Liu HK, Lin LC, Liou KT, Huang YC, Lin CH, Tzeng TT, Shie FS, Tsay HJ, Shiao YJ: Xuefu Zhuyu decoction ameliorates obesity, hepatic steatosis, neuroinflammation, amyloid deposition and cognition impairment in metabolically stressed APPswe/PS1dE9 mice. J Ethnopharmacol 2017, 209:50-61.

14. Yu G, Wang LG, Han Y, He QY: clusterProfiler: an R package for comparing biological themes among gene clusters. OMICS 2012, 16(5):284-287.

15. Lee WH, Jin JS, Tsai WC, Chen YT, Chang WL, Yao CW, Sheu LF, Chen A: Biological inhibitory effects of the Chinese herb danggui on brain astrocytoma. Pathobiology 2006, 73(3):141-148.

16. Barrett T, Wilhite SE, Ledoux P, Evangelista C, Kim IF, Tomashevsky M, Marshall KA, Phillippy KH, Sherman PM, Holko M et al: NCBI GEO: archive for functional genomics data sets-update. Nucleic Acids Res 2013, 41(Database issue):D991-995.

17. Guan D, Shao J, Zhao Z, Wang P, Qin J, Deng Y, Boheler KR, Wang J, Yan B: PTHGRN: unraveling post-translational hierarchical gene regulatory networks using PPI, ChIP-seq and gene expression data. Nucleic Acids Res 2014, 42(Web Server issue):W130-136.

18. Liu X, Vogt I, Haque T, Campillos M: HitPick: a web server for hit identification and target prediction of chemical screenings. Bioinformatics 2013, 29(15):1910-1912.

19. Tao W, Xu X, Wang X, Li B, Wang Y, Li Y, Yang L: Network pharmacology-based prediction of the active ingredients and potential targets of Chinese herbal Radix Curcumae formula for application to cardiovascular disease. J Ethnopharmacol 2013, 145(1):1-10.

20. Szklarczyk D, Santos A, von Mering C, Jensen LJ, Bork P, Kuhn M: STITCH 5: augmenting protein-chemical interaction networks with tissue and affinity data. Nucleic Acids Res 2016, 44(D1):D380-384.

21. Gfeller D, Grosdidier A, Wirth M, Daina A, Michielin O, Zoete V: SwissTargetPrediction: a web server for target prediction of bioactive small molecules. Nucleic Acids Res 2014, 42(Web Server issue):W32-38.

22. Lopes CT, Franz M, Kazi F, Donaldson SL, Morris Q, Bader GD: Cytoscape Web: an interactive web-based network browser. Bioinformatics 2010, 26(18):2347-2348.

23. de Jong H, Geiselmann J, Hernandez C, Page M: Genetic Network Analyzer: qualitative simulation of genetic regulatory networks. Bioinformatics 2003, 19(3):336-344.

24. Zeng X, Zhao J, Wu X, Shi H, Liu W, Cui B, Yang L, Ding X, Song P: PageRank analysis reveals topologically expressed genes correspond to psoriasis and their functions are associated with apoptosis resistance. Mol Med Rep 2016, 13(5):3969-3976.

25. Zhao J, Zeng X, Song P, Wu X, Shi H: AKT1 as the PageRank hub gene is associated with melanoma and its functional annotation is highly related to the estrogen signaling pathway that may regulate the growth of melanoma. Oncol Rep 2016, 36(4):2087-2093.

26. Draghici S, Khatri P, Tarca AL, Amin K, Done A, Voichita C, Georgescu C, Romero R: A systems biology approach for pathway level analysis. Genome Res 2007, 17(10):1537-1545.

27. Newman WC, Kim WJ, Amankulor NM: BRCA1-Regulated RRM2 Expression Protects Glioblastoma Cells from Endogenous Replication Stress and Promotes Tumorigenicity. Neurosurgery 2017, 80(5):N34.

28. Arivazhagan A, Kumar DM, Sagar V, Patric IR, Sridevi S, Thota B, Srividya MR, Prasanna K, Thennarasu K, Mondal N et al: Higher topoisomerase 2 alpha gene transcript levels predict better prognosis in GBM patients receiving temozolomide chemotherapy: identification of temozolomide as a TOP2A inhibitor. J Neurooncol 2012, 107(2):289-297.

29. Marie SK, Oba-Shinjo SM, da Silva R, Gimenez M, Nunes Reis G, Tassan JP, Rosa JC, Uno M: Stathmin involvement in the maternal embryonic leucine zipper kinase pathway in glioblastoma. Proteome Sci 2016, 14:6.

30. Qian Z, Li Y, Ma J, Xue Y, Xi Y, Hong L, Dai X, Zhang Y, Ji X, Chen Y et al: Prognostic value of NUSAP1 in progression and expansion of glioblastoma multiforme. J Neurooncol 2018, 140(2):199-208.

31. Zuo Z, Hu H, Xu Q, Luo X, Peng D, Zhu K, Zhao Q, Xie Y, Ren J: BBCancer: an expression atlas of blood-based biomarkers in the early diagnosis of cancers. Nucleic Acids Res 2020, 48(D1):D789-D796.

32. Hong-Yu YU, Yan-Qing LI, Li-Xiong C, Jun Z, Xue-Ying L, Ting-Bo C, Wen-Zhou LIJPT: Simultaneous Determination Content of Three Main Components in Xuefu Zhuyu Decoction by HPLC. Pharmacy Today 2009.

33. Huicheng YE, Qixin YE, Daimei W, Liyun ZJTCDR, Pharmacology C: HPLC Determination of Ferulic Acid in Xuefu Zhuyu Decoction. Traditional Chinese Drug Research \& Clinical Pharmacology 2009.

34. Li W, Qi Z, Wei Z, Liu S, Wang P, Chen Y, Zhao Y: Paeoniflorin inhibits proliferation and induces apoptosis of human glioma cells via microRNA-16 upregulation and matrix metalloproteinase-9 downregulation. Mol Med Rep 2015, 12(2):2735-2740.

35. Ouyang J, Xu H, Li M, Dai X, Fu F, Zhang X, Lan Q: Paeoniflorin exerts antitumor effects by inactivating S phase kinase-associated protein 2 in glioma cells. Oncol Rep 2018, 39(3):1052-1062.

Page 9/18 
36. Wang Z, Liu Z, Yu G, Nie X, Jia W, Liu RE, Xu R: Paeoniflorin Inhibits Migration and Invasion of Human Glioblastoma Cells via Suppression Transforming Growth Factor beta-Induced Epithelial-Mesenchymal Transition. Neurochem Res 2018, 43(3):760-774.

37. Hsu SS, Chou CT, Liao WC, Shieh P, Kuo DH, Kuo CC, Jan CR, Liang WZ: The effect of gallic acid on cytotoxicity, Ca(2+) homeostasis and ROS production in DBTRG-05MG human glioblastoma cells and CTX TNA2 rat astrocytes. Chem Biol Interact 2016, 252:61-73.

38. Lu Y, Jiang F, Jiang H, Wu K, Zheng X, Cai Y, Katakowski M, Chopp M, To SS: Gallic acid suppresses cell viability, proliferation, invasion and angiogenesis in human glioma cells. Eur J Pharmacol 2010, 641(2-3):102-107.

39. Paolini A, Curti V, Pasi F, Mazzini G, Nano R, Capelli E: Gallic acid exerts a protective or an anti-proliferative effect on glioma T98G cells via dosedependent epigenetic regulation mediated by miRNAs. Int J Oncol 2015, 46(4):1491-1497.

40. Tseng TH, Lee YJ: Evaluation of natural and synthetic compounds from East Asiatic folk medicinal plants on the mediation of cancer. Anticancer Agents Med Chem 2006, 6(4):347-365.

41. Belkaid A, Currie JC, Desgagnes J, Annabi B: The chemopreventive properties of chlorogenic acid reveal a potential new role for the microsomal glucose-6phosphate translocase in brain tumor progression. Cancer Cell Int 2006, 6:7.

42. Huang S, Wang LL, Xue NN, Li C, Guo HH, Ren TK, Zhan Y, Li WB, Zhang J, Chen XG et al: Chlorogenic acid effectively treats cancers through induction of cancer cell differentiation. Theranostics 2019, 9(23):6745-6763.

43. Xue N, Zhou Q, Ji M, Jin J, Lai F, Chen J, Zhang M, Jia J, Yang H, Zhang J et al: Chlorogenic acid inhibits glioblastoma growth through repolarizating macrophage from M2 to M1 phenotype. Sci Rep 2017, 7:39011.

44. Boyd NH, Walker K, Fried J, Hackney JR, McDonald PC, Benavides GA, Spina R, Audia A, Scott SE, Libby CJ et al: Addition of carbonic anhydrase 9 inhibitor SLC-0111 to temozolomide treatment delays glioblastoma growth in vivo. JCl Insight 2017, 2(24).

45. Tomar VS, Patil V, Somasundaram K: Temozolomide induces activation of Wnt/beta-catenin signaling in glioma cells via PI3K/Akt pathway: implications in glioma therapy. Cell Biol Toxicol 2020, 36(3):273-278.

46. Xia X, Li X, Li F, Wu X, Zhang M, Zhou H, Huang N, Yang X, Xiao F, Liu D et al: Correction to: A novel tumor suppressor protein encoded by circular AKT3 RNA inhibits glioblastoma tumorigenicity by competing with active phosphoinositide-dependent Kinase-1. Mol Cancer 2019, 18(1):149.

47. Kalhori MR, Irani S, Soleimani M, Arefian E, Kouhkan F: The effect of miR-579 on the PI3K/AKT pathway in human glioblastoma PTEN mutant cell lines. J Cell Biochem 2019, 120(10):16760-16774.

48. Stokoe D, Stephens LR, Copeland T, Gaffney PR, Reese CB, Painter GF, Holmes AB, McCormick F, Hawkins PT: Dual role of phosphatidylinositol-3,4,5trisphosphate in the activation of protein kinase B. Science 1997, 277(5325):567-570.

49. Arita H, Yamasaki K, Matsushita Y, Nakamura T, Shimokawa A, Takami H, Tanaka S, Mukasa A, Shirahata M, Shimizu S et al: A combination of TERT promoter mutation and MGMT methylation status predicts clinically relevant subgroups of newly diagnosed glioblastomas. Acta Neuropathol Commun 2016, 4(1):79.

50. Han L, Zhang K, Shi Z, Zhang J, Zhu J, Zhu S, Zhang A, Jia Z, Wang G, Yu S et al: LncRNA pro fi le of glioblastoma reveals the potential role of IncRNAs in contributing to glioblastoma pathogenesis. Int J Oncol 2012, 40(6):2004-2012.

51. Li X, Martinez-Ledesma E, Zhang C, Gao F, Zheng S, Ding J, Wu S, Nguyen N, Clifford SC, Wen PY et al: Tie2-FGFR1 Interaction Induces Adaptive PI3K Inhibitor Resistance by Upregulating Aurora A/PLK1/CDK1 Signaling in Glioblastoma. Cancer Res 2019, 79(19):5088-5101.

52. Cataltepe O, Arikan MC, Ghelfi E, Karaaslan C, Ozsurekci Y, Dresser K, Li Y, Smith TW, Cataltepe S: Fatty acid binding protein 4 is expressed in distinct endothelial and non-endothelial cell populations in glioblastoma. Neuropathol Appl Neurobiol 2012, 38(5):400-410.

53. Malmstrom A, Lysiak M, Akesson L, Jakobsen I, Mudaisi M, Milos P, Hallbeck M, Fomichov V, Broholm H, Grunnet K et al: ABCB1 single-nucleotide variants and survival in patients with glioblastoma treated with radiotherapy concomitant with temozolomide. Pharmacogenomics J 2020, 20(2):213-219.

54. Mete M, Unsal UU, Aydemir I, Sonmez PK, Tuglu MI: Punicic Acid Inhibits Glioblastoma Migration and Proliferation via the PI3K/AKT1/mTOR Signaling Pathway. Anticancer Agents Med Chem 2019, 19(9):1120-1131.

55. Wen N, Guo B, Zheng H, Xu L, Liang H, Wang Q, Wang D, Chen X, Zhang S, Li Y et al: Bromodomain inhibitor jq1 induces cell cycle arrest and apoptosis of glioma stem cells through the VEGF/PI3K/AKT signaling pathway. Int J Oncol 2019, 55(4):879-895.

56. Liu J, Lahousse L, Nivard MG, Bot M, Duijn CMVJNM: Integration of epidemiologic, pharmacologic, genetic and gut microbiome data in a drugmetabolite atlas. 2020, 26(1):110-117.

57. Basith S, Manavalan B, Hwan Shin T, Lee G: Machine intelligence in peptide therapeutics: A next-generation tool for rapid disease screening. Med Res Rev 2020, 40(4):1276-1314.

\section{Tables}

Table 1 The Information of chemical components in XFZYD. 


\begin{tabular}{|c|c|c|c|c|}
\hline Formula & Method & Component & Concentration & Ref. \\
\hline \multirow[t]{10}{*}{ Xuefu Zhuyu Decoction (XFZYD) } & \multirow[t]{10}{*}{ HPLC } & Hydroxy safflower yillow A & $0.7826 \mathrm{mg} / \mathrm{g}$ & \multirow[t]{3}{*}{ Yu et al., 2009} \\
\hline & & paeoniforin & $2.4205 \mathrm{mg} / \mathrm{g}$ & \\
\hline & & aringin & $2.4518 \mathrm{mg} / \mathrm{g}$ & \\
\hline & & Ferulic acid & $0.0751 \mathrm{mg} / \mathrm{g}$ & Ye et al., 2009 \\
\hline & & Gallic acid & $0.1078 \mathrm{mg} / \mathrm{g}$ & \multirow[t]{6}{*}{ Zhang et al., 2010} \\
\hline & & Protocatechuic acid & $0.1129 \mathrm{mg} / \mathrm{g}$ & \\
\hline & & Vanlillic acid & $0.0511 \mathrm{mg} / \mathrm{g}$ & \\
\hline & & Caffeic acid & $0.0203 \mathrm{mg} / \mathrm{g}$ & \\
\hline & & Chlorogenic acid & $0.0369 \mathrm{mg} / \mathrm{g}$ & \\
\hline & & Ferulic acid & $0.1021 \mathrm{mg} / \mathrm{g}$ & \\
\hline
\end{tabular}

Table 2 The active components information in XFZYD after ADMET screening. 


\begin{tabular}{|c|c|c|c|c|c|c|c|c|c|c|c|}
\hline ID & molecule_name & $\mathrm{OB}$ & DL & BBB & MW & ID & molecule_name & $\mathrm{OB}$ & $\mathrm{DL}$ & BBB & $\Lambda$ \\
\hline MOL000085 & beta-daucosterol_qt & 36.91 & 0.75 & 0.88 & 414.79 & MOL004810 & glyasperin $\mathrm{F}$ & 75.84 & 0.54 & -0.15 & 3 \\
\hline MOL000173 & wogonin & 30.68 & 0.23 & 0.04 & 284.28 & MOL004811 & Glyasperin C & 45.56 & 0.40 & 0.07 & 3 \\
\hline MOL000211 & Mairin & 55.38 & 0.78 & 0.22 & 456.78 & MOL004814 & Isotrifoliol & 31.94 & 0.42 & -0.25 & 2 \\
\hline MOL000239 & Jaranol & 50.83 & 0.29 & -0.22 & 314.31 & MOL004815 & $\begin{array}{l}\text { (E)-1-(2,4- } \\
\text { dihydroxyphenyl)-3-(2,2- } \\
\text { dimethylchromen-6- } \\
\text { yl)prop-2-en-1-one }\end{array}$ & 39.62 & 0.35 & -0.12 & 3 \\
\hline MOL000296 & hederagenin & 36.91 & 414.79 & 8.08 & 1.32 & MOL004820 & kanzonols W & 50.48 & 0.52 & 0.04 & 3 \\
\hline MOL000358 & beta-sitosterol & 36.91 & 0.75 & 0.99 & 414.79 & MOL004828 & Glepidotin A & 44.72 & 0.35 & 0.06 & 3 \\
\hline MOL000359 & sitosterol & 36.91 & 0.75 & 0.87 & 414.79 & MOL004829 & Glepidotin B & 64.46 & 0.34 & -0.09 & 3 \\
\hline MOL000392 & formononetin & 69.67 & 0.21 & 0.02 & 268.28 & MOL004833 & Phaseolinisoflavan & 32.01 & 0.45 & 0.46 & 3 \\
\hline MOL000432 & linolenic acid & 45.01 & 0.15 & 0.84 & 278.48 & MOL004835 & Glypallichalcone & 61.60 & 0.19 & 0.23 & 2 \\
\hline MOL000449 & Stigmasterol & 43.83 & 0.76 & 1.00 & 412.77 & MOL004838 & $\begin{array}{l}\text { 8-(6-hydroxy-2- } \\
\text { benzofuranyl)-2,2- } \\
\text { dimethyl-5-chromenol }\end{array}$ & 58.44 & 0.38 & 0.34 & 3 \\
\hline MOL000493 & campesterol & 37.58 & 400.76 & 7.63 & 1.31 & MOL004848 & licochalcone G & 49.25 & 0.32 & -0.04 & 3 \\
\hline MOL000497 & licochalcone a & 40.79 & 0.29 & -0.21 & 338.43 & MOL004849 & $\begin{array}{l}\text { 3-(2,4- } \\
\text { dihydroxyphenyl)-8-(1,1- } \\
\text { dimethylprop-2-enyl)-7- } \\
\text { hydroxy-5-methoxy- } \\
\text { coumarin }\end{array}$ & 59.62 & 0.43 & -0.23 & 3 \\
\hline MOL000500 & Vestitol & 74.66 & 0.21 & 0.30 & 272.32 & MOL004855 & Licoricone & 63.58 & 0.47 & -0.14 & 3 \\
\hline MOL000785 & palmatine & 64.60 & 0.65 & 0.37 & 352.44 & MOL004856 & Gancaonin A & 51.08 & 0.40 & 0.13 & 3 \\
\hline MOL000953 & CLR & 37.87 & 0.68 & 1.13 & 386.73 & MOL004857 & Gancaonin B & 48.79 & 0.45 & -0.10 & 3 \\
\hline MOL001006 & $\begin{array}{l}\text { poriferasta-7,22E-dien- } \\
\text { 3beta-ol }\end{array}$ & 42.98 & 0.76 & 1.11 & 412.77 & MOL004863 & $\begin{array}{l}\text { 3-(3,4- } \\
\text { dihydroxyphenyl)-5,7- } \\
\text { dihydroxy-8-(3- } \\
\text { methylbut-2- } \\
\text { enyl)chromone }\end{array}$ & 66.37 & 0.41 & -0.13 & 3 \\
\hline MOL001323 & Sitosterol alpha1 & 43.28 & 426.80 & 8.15 & 1.41 & MOL004864 & $\begin{array}{l}\text { 5,7-dihydroxy-3-(4- } \\
\text { methoxyphenyl)-8-(3- } \\
\text { methylbut-2- } \\
\text { enyl)chromone }\end{array}$ & 30.49 & 0.41 & 0.21 & 3 \\
\hline MOL001340 & GA120 & 84.85 & 314.41 & 2.47 & 0.38 & MOL004866 & $\begin{array}{l}\text { 2-(3,4- } \\
\text { dihydroxyphenyl)-5,7- } \\
\text { dihydroxy-6-(3- } \\
\text { methylbut-2- } \\
\text { enyl)chromone }\end{array}$ & 44.15 & 0.41 & -0.28 & 3 \\
\hline MOL001398 & Methyllinolenate & 46.15 & 0.17 & 1.09 & 292.51 & MOL004879 & Glycyrin & 52.61 & 0.47 & -0.13 & 3 \\
\hline MOL001454 & berberine & 36.86 & 0.78 & 0.57 & 336.39 & MOL004882 & Licocoumarone & 33.21 & 0.36 & 0.06 & 3 \\
\hline MOL001458 & coptisine & 30.67 & 0.86 & 0.32 & 320.34 & MOL004883 & Licoisoflavone & 41.61 & 0.42 & -0.27 & 3 \\
\hline MOL001484 & Inermine & 75.18 & 0.54 & 0.40 & 284.28 & MOL004884 & Licoisoflavone B & 38.93 & 0.55 & -0.18 & 3 \\
\hline MOL001494 & Mandenol & 42.00 & 0.19 & 1.14 & 308.56 & MOL004885 & licoisoflavanone & 52.47 & 0.54 & -0.22 & 3 \\
\hline MOL001645 & Linoleyl acetate & 42.10 & 0.20 & 1.08 & 308.56 & MOL004891 & shinpterocarpin & 80.30 & 0.73 & 0.68 & 3 \\
\hline MOL001689 & acacetin & 34.97 & 0.24 & -0.05 & 284.28 & MOL004907 & Glyzaglabrin & 61.07 & 0.35 & -0.20 & 2 \\
\hline MOL001771 & poriferast-5-en-3beta-ol & 36.91 & 0.75 & 1.14 & 414.79 & MOL004908 & Glabridin & 53.25 & 0.47 & 0.36 & 3 \\
\hline MOL001792 & DFV & 32.76 & 0.18 & -0.29 & 256.27 & MOL004910 & Glabranin & 52.90 & 0.31 & 0.31 & 3 \\
\hline MOL002135 & Myricanone & 40.60 & 0.51 & -0.08 & 356.45 & MOL004911 & Glabrene & 46.27 & 0.44 & 0.04 & 3 \\
\hline MOL002140 & Perlolyrine & 65.95 & 0.27 & 0.15 & 264.30 & MOL004912 & Glabrone & 52.51 & 0.50 & -0.11 & 3 \\
\hline MOL002151 & senkyunone & 47.66 & 0.24 & 0.50 & 326.52 & MOL004913 & $\begin{array}{l}\text { 1,3-dihydroxy-9-methoxy- } \\
\text { 6-benzofurano[3,2- } \\
\text { c]chromenone }\end{array}$ & 48.14 & 0.43 & -0.19 & 2 \\
\hline MOL002157 & wallichilide & 42.31 & 0.71 & 0.73 & 412.57 & MOL004915 & Eurycarpin A & 43.28 & 0.37 & -0.06 & 3 \\
\hline MOL002311 & Glycyrol & 90.78 & 0.67 & -0.20 & 366.39 & MOL004941 & $\begin{array}{l}\text { (2R)-7-hydroxy-2-(4- } \\
\text { hydroxyphenyl)chroman- }\end{array}$ & 71.12 & 0.18 & -0.25 & 2 \\
\hline
\end{tabular}




\begin{tabular}{|c|c|c|c|c|c|c|c|c|c|c|c|}
\hline & & & & & & & 4-one & & & & \\
\hline MOL002341 & Hesperetin & 70.31 & 0.27 & -0.25 & 302.30 & MOL004945 & $\begin{array}{l}\text { (2S)-7-hydroxy-2-(4- } \\
\text { hydroxyphenyl)-8-(3- } \\
\text { methylbut-2- } \\
\text { enyl)chroman-4-one }\end{array}$ & 36.57 & 0.32 & -0.04 & 3 \\
\hline MOL002565 & Medicarpin & 49.22 & 0.34 & 0.53 & 270.30 & MOL004948 & Isoglycyrol & 44.70 & 0.84 & 0.05 & 3 \\
\hline MOL002643 & delta 7-stigmastenol & 37.42 & 0.75 & 0.83 & 414.79 & MOL004957 & HMO & 38.37 & 0.21 & 0.25 & 2 \\
\hline MOL002695 & lignan & 43.32 & 0.65 & -0.16 & 458.55 & MOL004959 & 1-Methoxyphaseollidin & 69.98 & 0.64 & 0.48 & 3 \\
\hline MOL002698 & lupeol-palmitate & 33.98 & 0.32 & 0.89 & 665.26 & MOL004966 & $\begin{array}{l}\text { 3'-Hydroxy-4'-0- } \\
\text { Methylglabridin }\end{array}$ & 43.71 & 0.57 & 0.73 & 3 \\
\hline MOL002706 & Phytoene & 39.56 & 0.50 & 1.70 & 545.04 & MOL004974 & 3'-Methoxyglabridin & 46.16 & 0.57 & 0.47 & 3 \\
\hline MOL002707 & phytofluene & 43.18 & 0.50 & 1.76 & 543.02 & MOL004978 & $\begin{array}{l}\text { 2-[(3R)-8,8-dimethyl-3,4- } \\
\text { dihydro-2H-pyrano[6,5- } \\
\text { f]chromen-3-yl]-5- } \\
\text { methoxyphenol }\end{array}$ & 36.21 & 0.52 & 0.61 & 3 \\
\hline MOL002710 & Pyrethrin II & 48.36 & 0.35 & -0.21 & 372.50 & MOL004980 & Inflacoumarin A & 39.71 & 0.33 & -0.24 & 3 \\
\hline MOL002714 & baicalein & 33.52 & 0.21 & -0.05 & 270.25 & MOL004985 & icos-5-enoic acid & 30.70 & 0.20 & 1.09 & 3 \\
\hline MOL002719 & 6-Hydroxynaringenin & 33.23 & 0.24 & -0.27 & 288.27 & MOL004988 & Kanzonol F & 32.47 & 0.89 & 0.56 & 4 \\
\hline MOL002773 & beta-carotene & 37.18 & 0.58 & 1.52 & 536.96 & MOL004989 & 6-prenylated eriodictyol & 39.22 & 0.41 & -0.29 & 3 \\
\hline MOL002897 & epiberberine & 43.09 & 0.78 & 0.40 & 336.39 & MOL004991 & $\begin{array}{l}\text { 7-Acetoxy-2- } \\
\text { methylisoflavone }\end{array}$ & 38.92 & 0.26 & 0.16 & 2 \\
\hline MOL003656 & Lupiwighteone & 51.64 & 0.37 & -0.23 & 338.38 & MOL004996 & gadelaidic acid & 30.70 & 0.20 & 0.94 & 3 \\
\hline MOL003847 & Inophyllum E & 38.81 & 0.85 & 0.30 & 402.47 & MOL005000 & Gancaonin G & 60.44 & 0.39 & 0.23 & 3 \\
\hline MOL003896 & $\begin{array}{l}\text { 7-Methoxy-2-methyl } \\
\text { isoflavone }\end{array}$ & 42.56 & 0.20 & 0.56 & 266.31 & MOL005001 & Gancaonin $\mathrm{H}$ & 50.10 & 0.78 & -0.14 & 4 \\
\hline MOL004355 & Spinasterol & 42.98 & 0.76 & 1.04 & 412.77 & MOL005003 & Licoagrocarpin & 58.81 & 0.58 & 0.61 & 3 \\
\hline MOL004598 & $\begin{array}{l}\text { 3,5,6,7-tetramethoxy-2- } \\
(3,4,5- \\
\text { trimethoxyphenyl)chromone }\end{array}$ & 31.97 & 0.59 & 0.08 & 432.46 & MOL005007 & Glyasperins M & 72.67 & 0.59 & -0.04 & 3 \\
\hline MOL004609 & Areapillin & 48.96 & 0.41 & -0.29 & 360.34 & MOL005012 & Licoagroisoflavone & 57.28 & 0.49 & 0.09 & 3 \\
\hline MOL004624 & Longikaurin A & 47.72 & 0.53 & 0.09 & 348.48 & MOL005016 & Odoratin & 49.95 & 0.30 & -0.24 & 3 \\
\hline MOL004628 & Octalupine & 47.82 & 0.28 & 0.30 & 264.41 & MOL005017 & Phaseol & 78.77 & 0.58 & -0.06 & 3 \\
\hline MOL004644 & Sainfuran & 79.91 & 0.23 & 0.23 & 286.30 & MOL005018 & Xambioona & 54.85 & 0.87 & 0.52 & 3 \\
\hline MOL004648 & Troxerutin & 31.60 & 0.28 & -0.38 & 346.56 & MOL005020 & dehydroglyasperins C & 53.82 & 0.37 & -0.12 & 3 \\
\hline MOL004653 & $(+)$-Anomalin & 46.06 & 0.66 & 0.00 & 426.50 & MOL005828 & nobiletin & 61.67 & 0.52 & -0.08 & 4 \\
\hline MOL004718 & a-spinasterol & 42.98 & 0.76 & 0.79 & 412.77 & MOL006999 & stigmast-7-en-3-ol & 37.42 & 0.75 & 0.85 & 4 \\
\hline MOL004805 & $\begin{array}{l}\text { (2S)-2-[4-hydroxy-3-(3- } \\
\text { methylbut-2- } \\
\text { enyl)phenyl]-8,8-dimethyl- } \\
\text { 2,3-dihydropyrano[2,3- } \\
\text { f]chromen-4-one }\end{array}$ & 31.79 & 0.72 & 0.25 & 390.51 & MOL007012 & $\begin{array}{l}\text { 4-o-methyl- } \\
\text { paeoniflorin_qt }\end{array}$ & 56.70 & 0.43 & -0.20 & 3 \\
\hline MOL004806 & euchrenone & 30.29 & 0.57 & 0.39 & 406.56 & MOL012461 & 28-norolean-17-en-3-ol & 35.93 & 0.78 & 1.12 & 4 \\
\hline MOL004808 & glyasperin B & 65.22 & 0.44 & -0.09 & 370.43 & & & & & & \\
\hline
\end{tabular}


Table 3 The information of CCG.

\begin{tabular}{llllll}
\hline ID & Name & MV & BBB & OB & DL \\
\hline MOL004996 & gadelaidic acid & 310.58 & 0.93932 & 30.70294255 & 0.19725 \\
MOL004985 & icos-5-enoic acid & 310.58 & 1.09146 & 30.70294255 & 0.19725 \\
MOL001689 & acacetin & 284.28 & -0.04689 & 34.97357273 & 0.24082 \\
MOL003896 & 7-Methoxy-2-methyl isoflavone & 266.31 & 0.55574 & 42.56474148 & 0.19946 \\
MOL004835 & Glypallichalcone & 284.33 & 0.22993 & 61.59706227 & 0.18993 \\
MOL000432 & linolenic acid & 278.48 & 0.83697 & 45.00906591 & 0.14709 \\
MOL000173 & wogonin & 284.28 & 0.03762 & 30.68456706 & 0.22942 \\
MOL000239 Jaranol & 1,3-dihydroxy-9-methoxy-6-benzofurano[3,2-c]chromenone & 314.31 & -0.21709 & 50.82881677 & 0.29148 \\
MOL004913 & 298.26 & -0.18646 & 48.14154235 & 0.42831 \\
MOL000392 & formononetin & 268.28 & 1.08413 & 42.10076623 & 0.19845 \\
MOL001645 & Linoleyl acetate & 308.56 & 1.08413 & 42.10076623 & 0.19845 \\
MOL004866 & 2-(3,4-dihydroxyphenyl)-5,7-dihydroxy-6-(3-methylbut-2-enyl)chromone & 354.38 & -0.27591 & 44.15196126 & 0.41482 \\
MOL004991 & 7-Acetoxy-2-methylisoflavone & 294.32 & 0.15501 & 38.92333105 & 0.26217 \\
MOL004814 & 2-(3,4-dihydroxyphenyl)-5,7-dihydroxy-6-(3-methylbut-2-enyl)chromone & 354.38 & 1.14299 & 36.91390583 & 0.75034 \\
MOL001771 & poriferast-5-en-3beta-ol & 414.79 & 1.14299 & 36.91390583 & 0.75034 \\
MOL001494 & Mandenol & 308.56 & 1.13821 & 41.99620045 & 0.19321 \\
MOL004644 & Sainfuran & 286.3 & 0.23234 & 79.90979196 & 0.23331 \\
MOL002714 & baicalein & 270.25 & -0.05023 & 33.51891869 & 0.20888 \\
MOL004855 Licoricone & 382.44 & -0.14145 & 63.57845938 & 0.4712 \\
MOL005017 & Phaseol & 336.36 & -0.05608 & 78.76621925 & 0.57867 \\
MOL004879 & Glycyrin & 382.44 & -0.12803 & 52.60657166 & 0.47466 \\
\hline
\end{tabular}

\section{Figures}

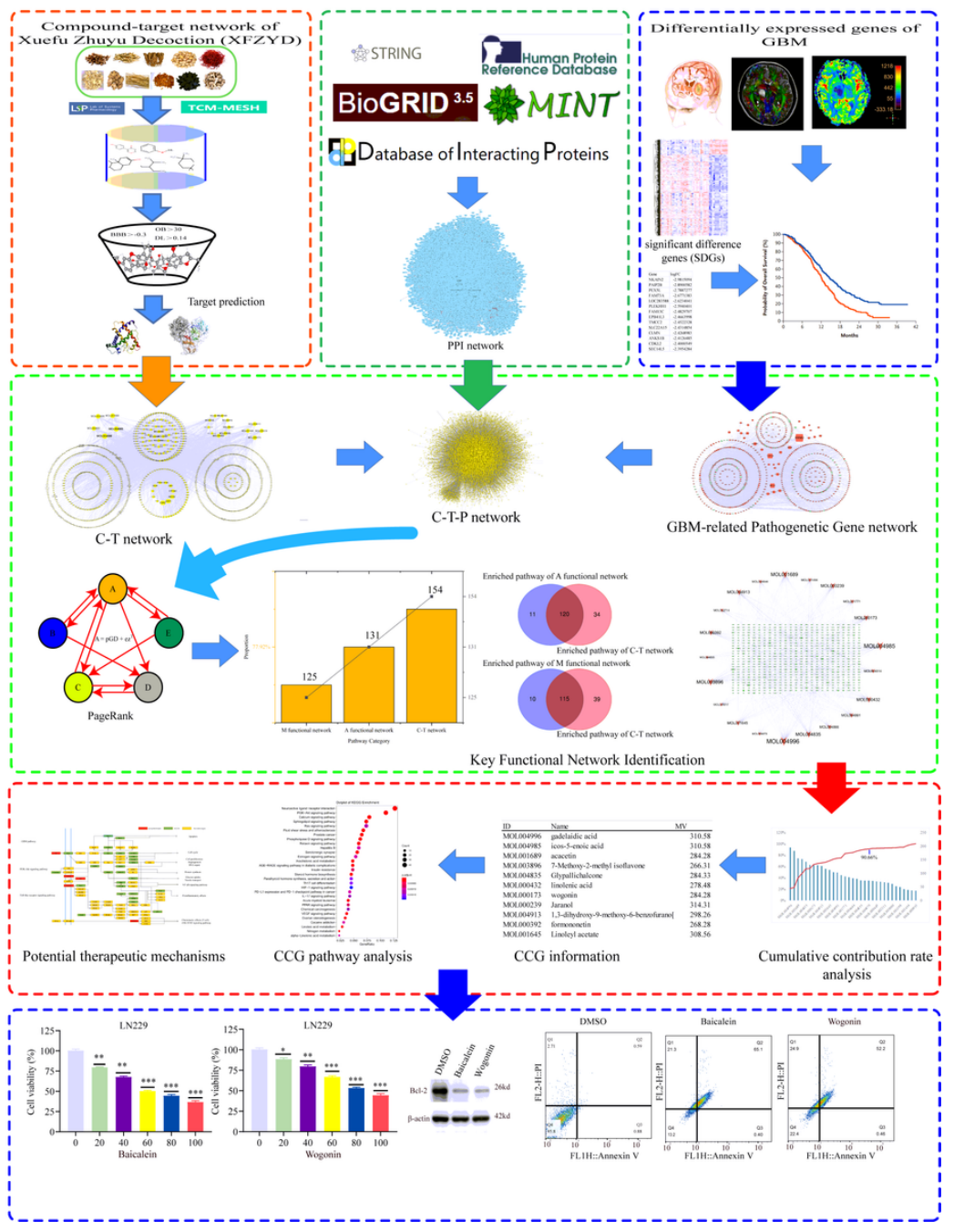

Figure 1

The schematic diagram of our proposed network pharmacology strategy. 


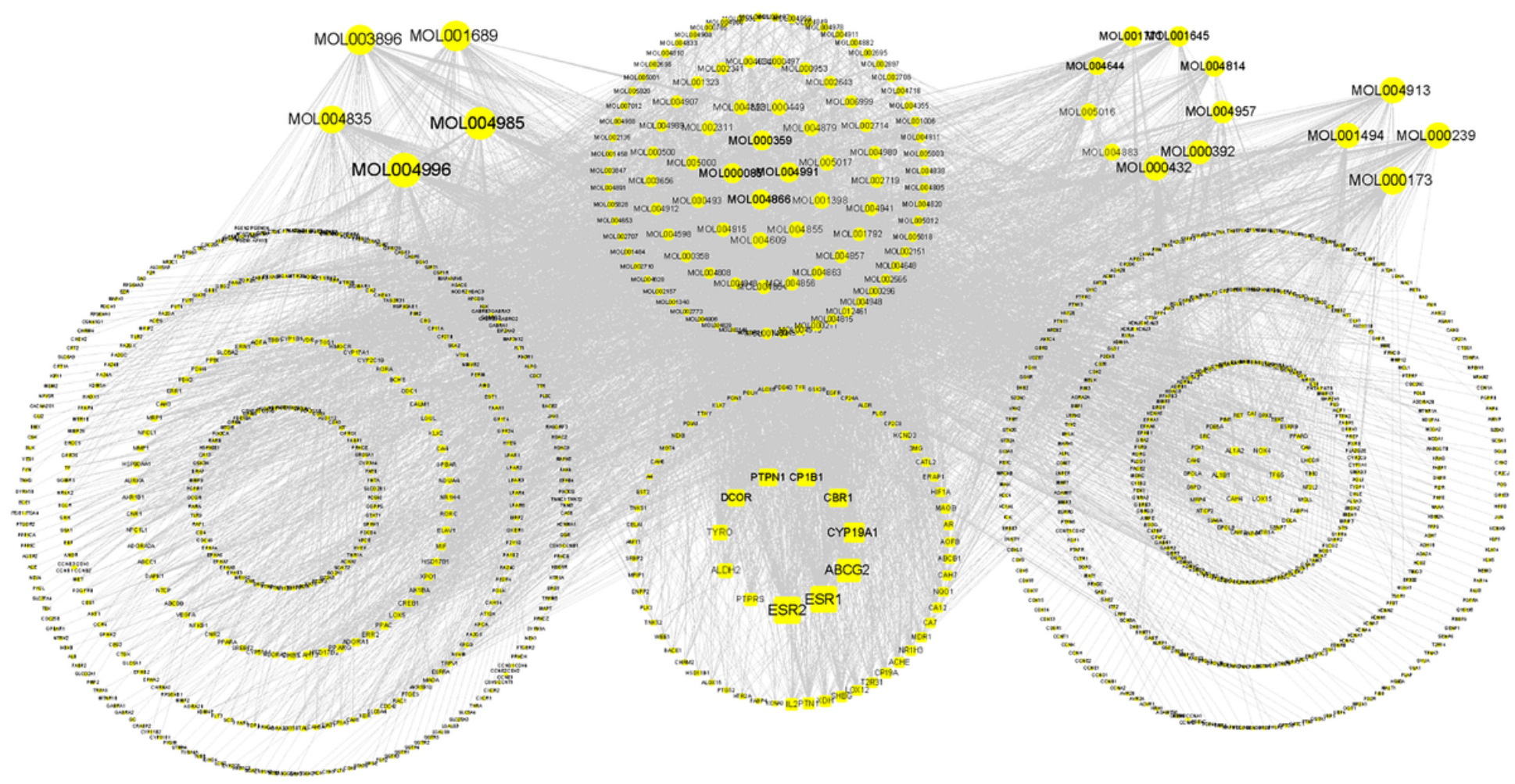

Figure 2

The C-T network of 117 active components and their targets. The component is represented by red and the target is represented by green.

A

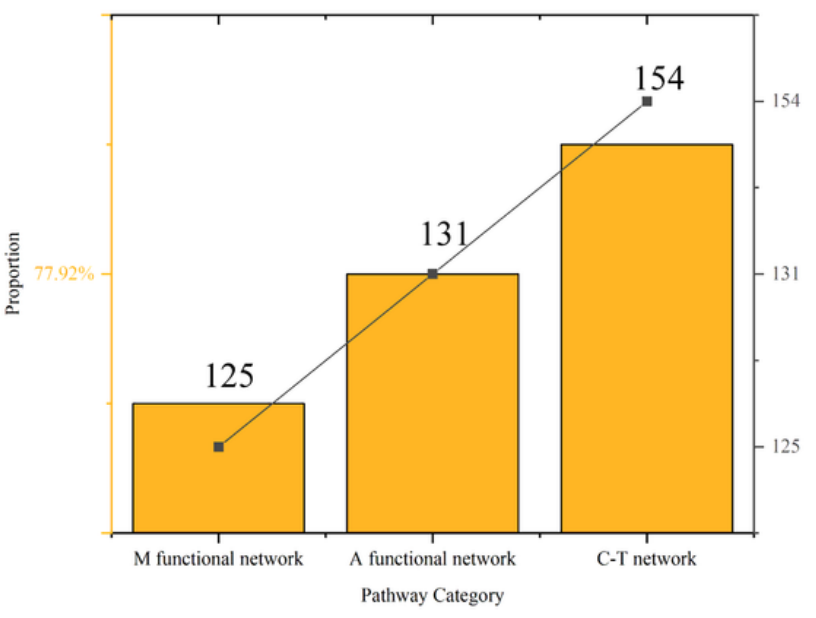

C

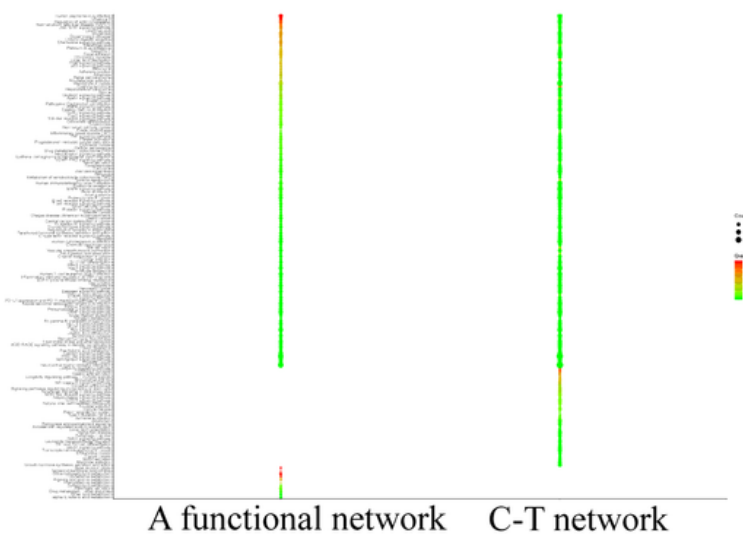

B

Enriched pathway of A functional network

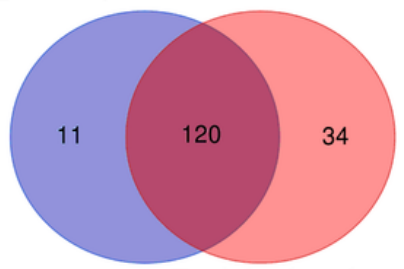

Enriched pathway of C-T network

Enriched pathway of $\mathrm{M}$ functional network

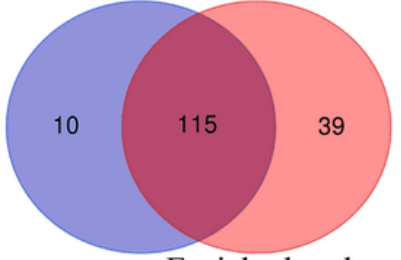

Enriched pathway of C-T network

D

$\overline{1}$

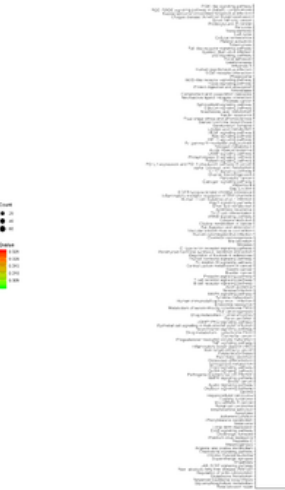

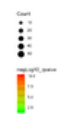

Page 15/18 
Figure 3

Determination of the key functional network. A: Histogram shows the gene enrichment pathway in A functional network and M functional network, accounting for the proportion of gene enrichment pathway in C-T network. B: Venn diagram shows the coincidence of gene enrichment pathways in A functional network, $M$ functional network and C-T network. C: Bubble diagram for common pathway enrichment analysis of A functional network and C-T network. D: Bubble diagram for common pathway enrichment analysis of A functional network and the differential expression genes.

$120 \%$

$100 \%$

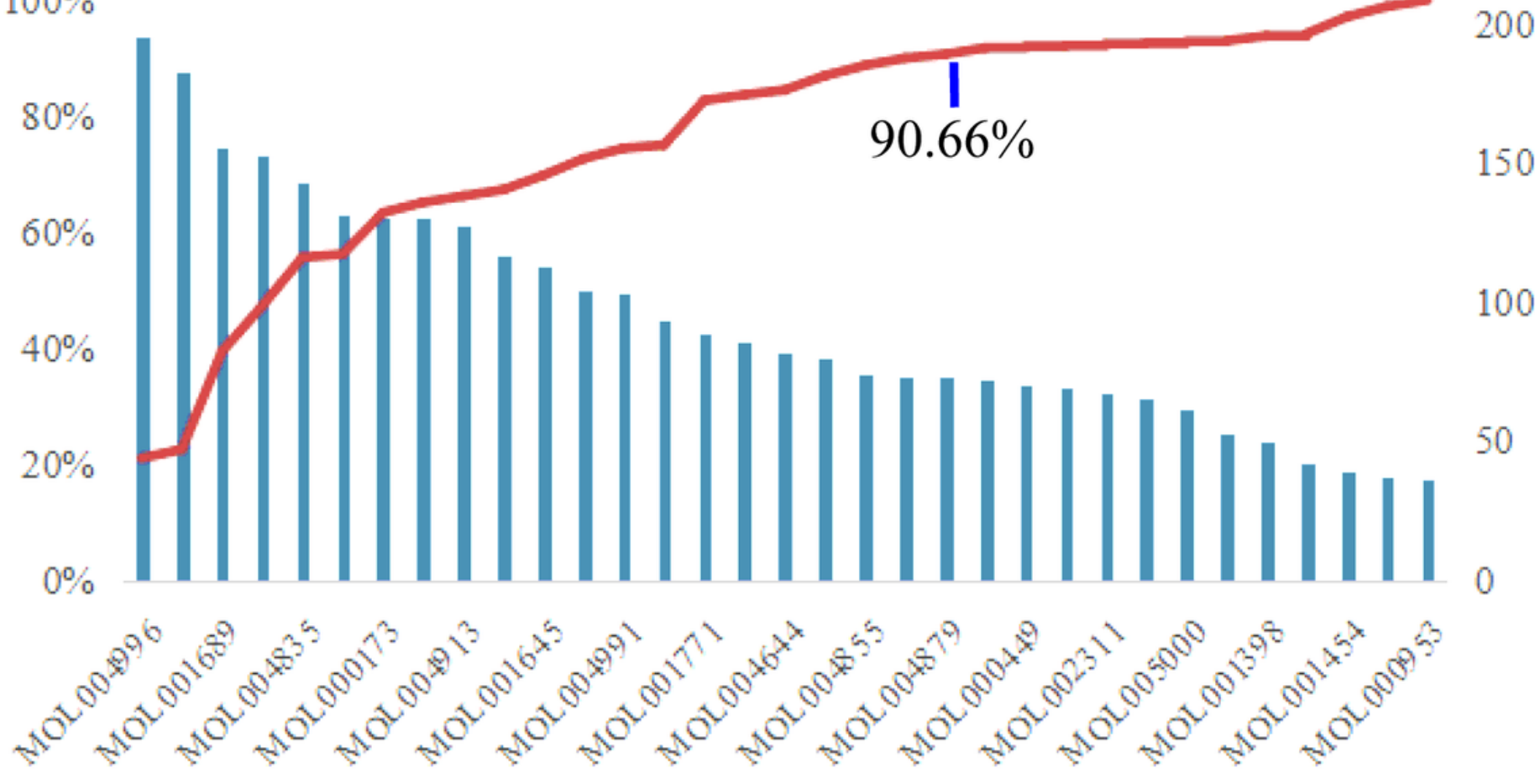

Figure 4

The CCR accumulation for CCG selection in XFZYD.

A

B

Enriched pathway of CCG

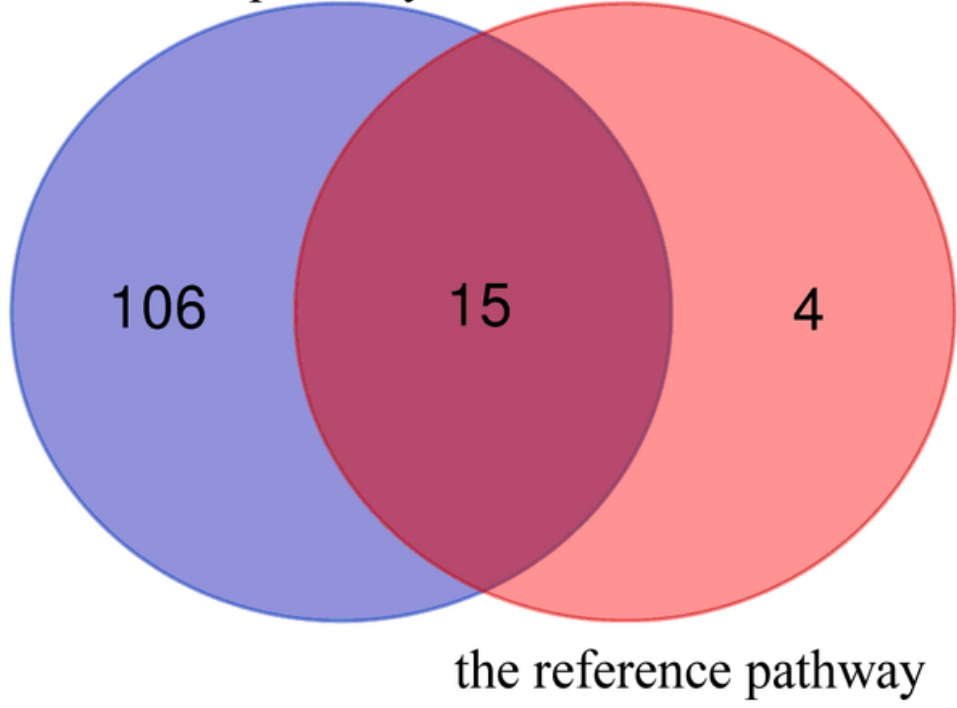

Dotplot of KEGG Enrichment

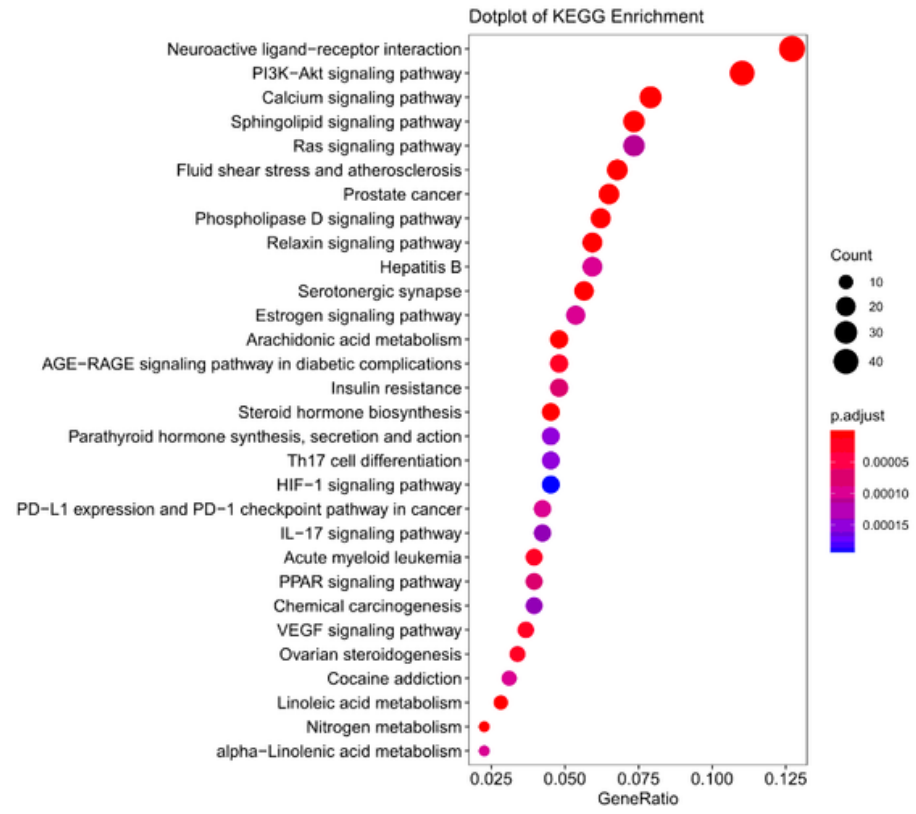




\section{Figure 5}

CCG Prediction and validation. A: Venn diagram shows the coincidence of gene enrichment pathways in CCG and the reference pathway. B: Bubble diagram for top 30 pathway enrichment of CCG targets.

A

$\mathrm{B}$

\section{$\mathrm{GO}$ analysis of $\mathrm{CCG}$}

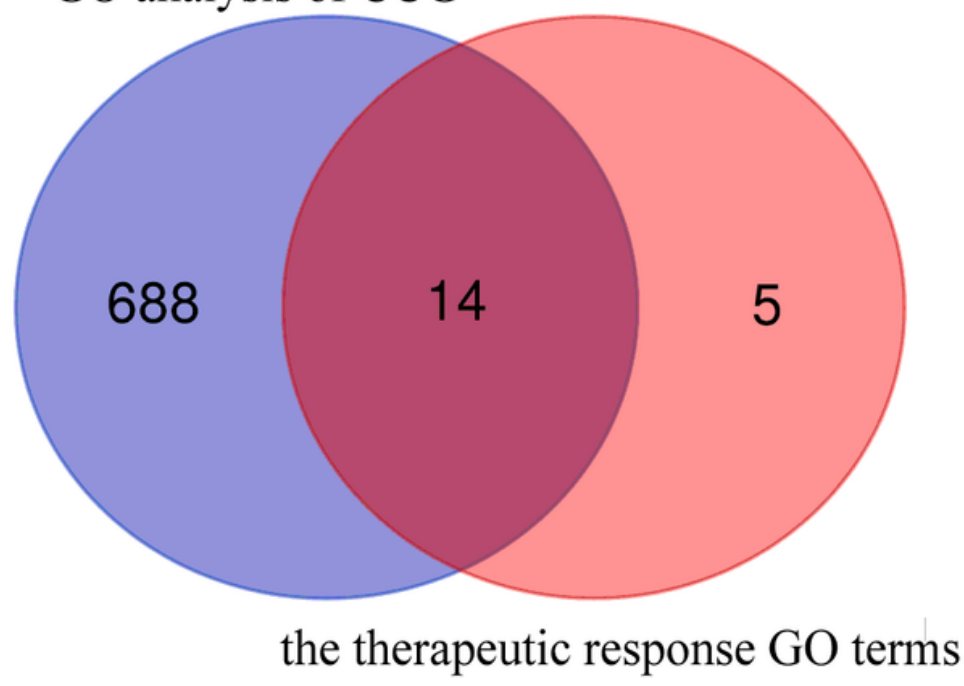

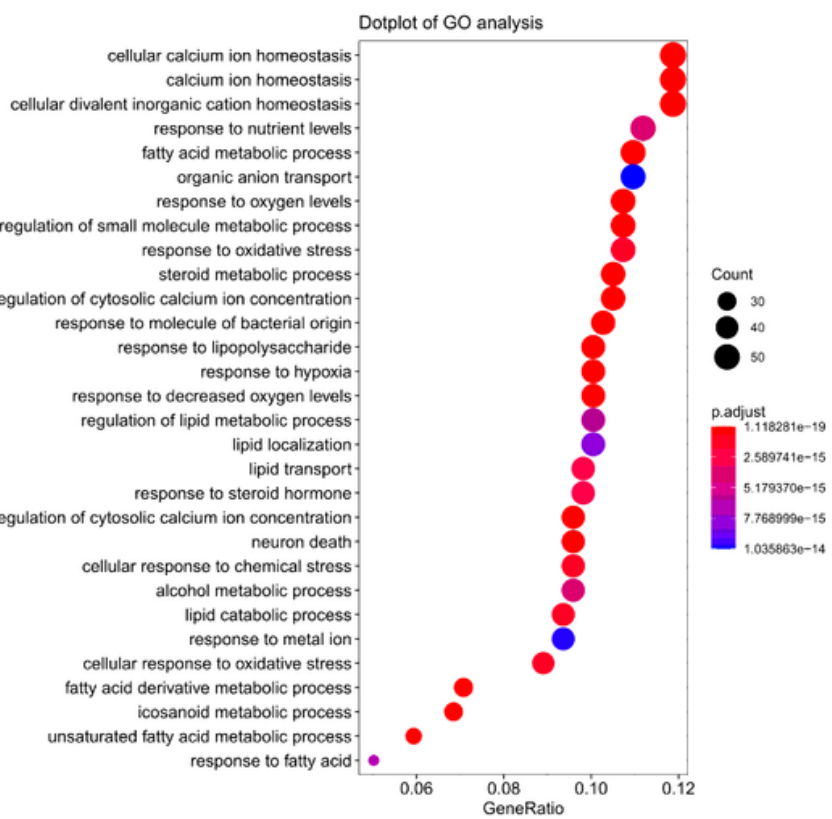

Figure 6

The biological processes analysis of CCG. A: Venn diagram shows the coincidence of GO analysis in CCG and the therapeutic response GO terms. B: Bubble diagram for top $30 \mathrm{GO}$ enrichment of CCG.

GBM pathway

PI3K-Akt signaling pathway

Toll-like receptor signaling pathway
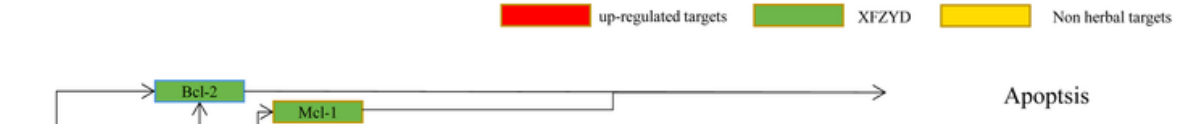

Cell cycle

Cell proliferation

Angiogenesis

DNA repair

Protein synthesis

Glucose uptake

Vesicle transport

$\mathrm{NF} \kappa \mathrm{B}$ signaling pathway

Proinflammatory effects

Chemotactic effects ( $T$ cell) JAK-STAT signaling pathway

Figure 7 
Distribution of CCG targets on the comprehensive signaling pathway.

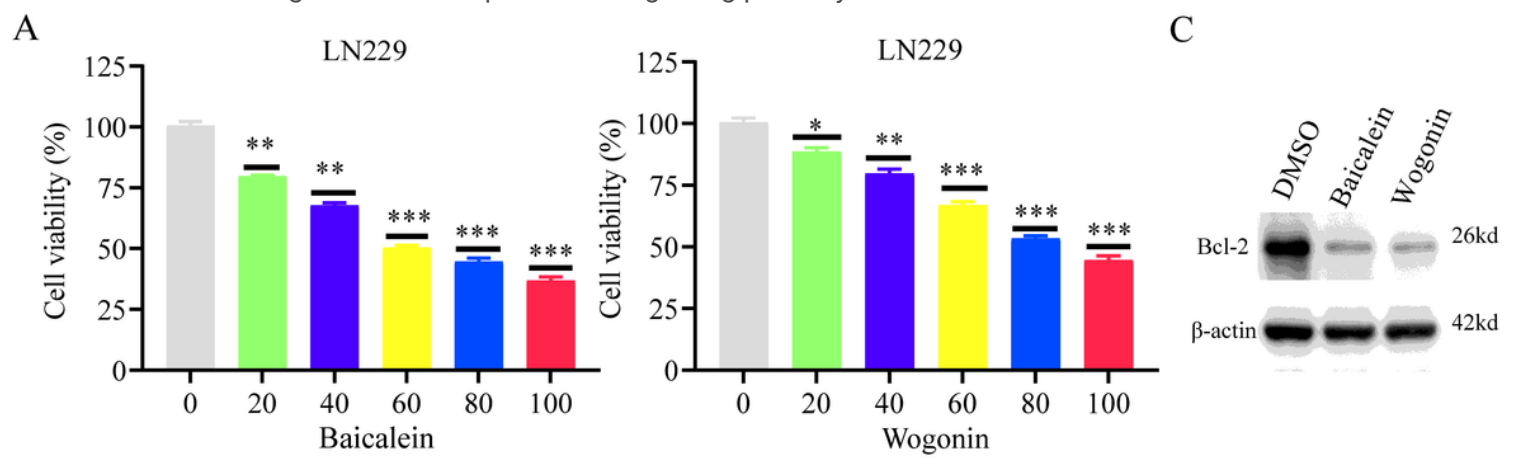

B

DMSO

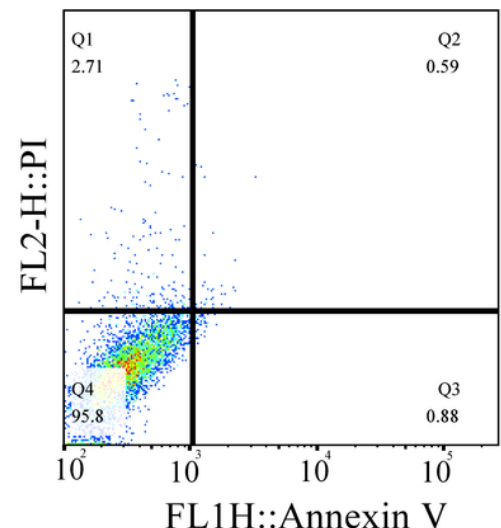

Baicalein

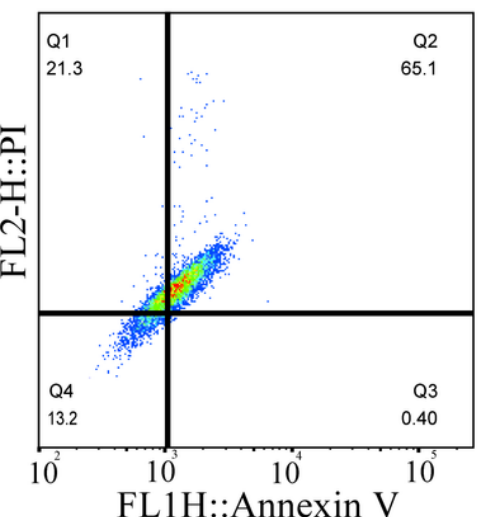

Wogonin

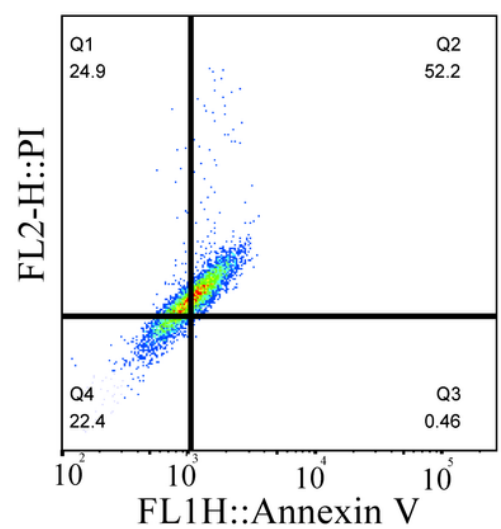

\section{Figure 8}

A: The effect of treatment with baicalein and wogonon on cell viabilities of LN229 cells ( $n=3)$. B: Effects of baicalein $(60 \mu M)$ and wogonon ( $80 \mu M)$ on LN229 cells apoptosis based on flow cytometry analyses $(n=3)$, C: Effects of baicalein $(60 \mu \mathrm{M})$ and wogonon $(80 \mu \mathrm{M})$ on Bcl-2 expression in LN229 cells.and reduced respectively $\mathrm{Bcl}-2$ protein expression. ${ }^{*} \mathrm{p}<0.05,{ }^{* \star} \mathrm{p}<0.01,{ }^{\star \star \star} \mathrm{p}<0.001$ versus control group.

\section{Supplementary Files}

This is a list of supplementary files associated with this preprint. Click to download.

- Supplementaryfigures1.tif

- Supplementaryfigures2.tif

- SupplementaryfigureS3.tif

- SupplementaryfigureS4.tif 